\title{
OPORTUNIDADES Y DESAFÍOS RELEVANTES DE LA EDUCACIÓN PARA LA COMUNICACIÓN CIUDADANA COMO CONCRECIÓN DE UNA AGENDA DE DESARROLLO LOCAL SOSTENIBLE
}

\author{
María Elena López León. Universidad de la Habana, Cuba.
}

Manuel Paulino Linares Herrera. Academia de ciencias de Cuba, Cuba.

Felisa Junco Martínez. Universidad de la Habana, Cuba.

\section{RESUMEN}

La concepción del uso de las TIC con el propósito de formar sujetos capaces de interactuar con ellas para el ejercicio de la crítica, la creatividad y la responsabilidad social. El empleo de La Educación para la Comunicación como principio rector, para comprender la relación entre los actores del proceso de comunicación (relación entre los productores, el proceso productivo, su producto y la recepción de los mensajes en circularidad constante), así como en el campo pedagógico, el asumido por los programas de formación de receptores autónomos y críticos frente a los medios digitales. El estudio del uso de las TIC desde un enfoque humanista sustentado en la teoría de las mediaciones sociales, como modelo de aprendizaje que sitúa al sujeto como centro del proceso de enseñanza aprendizaje (PEA), permitiendo lograr una formación cultural-cívico-pedagógico concebida a partir del principio de contribuir a la formación de un individuo con capacidades para transformar las estructuras y sistemas de comunicación en la sociedad. Es la razón de ser de presente artículo.

PALABRAS CLAVE: educación para la comunicación; mediaciones; comunicación para el cambio social; TIC.

León León, M. E., Linares Herrera, M. P. y Junco Martínez, F. (2018). Oportunidades y desafíos relevantes de la educación para la comunicación ciudadana como concreción de una agenda de desarrollo local sostenible. Revista de Ciencias de la Comunicación e Información, 23(2), 15-25.

doi: http://doi.org/10.35742/rcci.2018.23(2).15-25

\section{INTRODUCCIÓN}

Entre los años 2004 y 2018 diferentes autores internacionales y nacionales han contribuido a sustentar la importancia de la EPC en el empoderamiento ciudadano, desde la crítica a las concepciones dominantes sobre la participación ciudadana, asumiendo para ello una comprensión de las TIC como plataformas sociotécnicas integradas a su entorno político, económico y sociocultural. Proyectos extensionistas: Alma Mater y Escaramujo en Cuba y el Estudio de Caso: Comunidad Ómnia-Barrio el Raval, Barcelona del 2007 sirven como antecedentes a la investigación Educación para la Comunicación ciudadana mediada por las TIC. Propuesta de modelo de EPCc como contribución al desarrollo local. 
La existencia en Cuba de un desarrollo limitado de experiencias desde lo institucional en el uso de las TIC con una concepción de empleo humanística dirigida a formar sujetos capaces de interactuar y usar los recursos de la comunicación a partir del ejercicio de la crítica, la creatividad y la responsabilidad social.

La falta de políticas formales orientadas al trabajo educativo en función de los medios digitales, lo cual ha desarrollado experiencias de Educación para la Comunicación asociadas más al interés de las entidades que las conciben o implementan, que, de las necesidades propias de los sujetos, lo que trae como consecuencia una mediación institucional preponderante en la concepción e implementación de las experiencias de este tipo, en detrimento de la participación activa de los destinatarios/usuarios.

La falta de sistematicidad en las experiencias de Educación para la Comunicación ciudadana en los Joven Club de Computación y Electrónica como entidad mediadora en el uso de las TIC en sujetos de comunidades situación de vulnerabilidad social, cuyas prácticas adolecen de evaluación y análisis, que permita conocer sus fortalezas, debilidades, principales aportes y particularidades.

Y la ausencia de estrategias metodológicas que contribuyan al desarrollo de la Educación para la Comunicación en sujetos de comunidades en situación de vulnerabilidad en una institución como Joven Club de Computación y Electrónica que asume el trabajo educativo en función de los medios digitales propician entre otras el desarrollo de la investigación que se propone.

Se estaría hablando de una propuesta socioeducativa, la cual estaría sustentada en los principios de una cultura transformadora ${ }^{1}$ y que según Ibáñez (2004) actuaría como catalizador en el proceso de empoderamiento de los ciudadanos, contribuyendo a desarrollar niveles de comprensión sobre la realidad de la que forman parte, propiciando una actuación critica que contribuya a superar los niveles de pobreza y desigualdad, desde la experiencia vivida, al aportar desde su creatividad una participación en la contribución de soluciones alternativas.

Para ello es imprescindible el apoyo en el uso de las TIC desde una concepción de aprendizaje desarrollador ${ }^{2}$, (Castellanos, Reinoso, \& García, 2000), donde las tecnologías de la información y las comunicaciones, serían utilizadas como mediación, en un contexto educativo no formal como lo son los Joven Club de Computación y Electrónica a partir de la propuesta de Martínez de Toda (1998), para ayudar a la formación de un sujeto: alfabetizado mediáticamente; consciente; activo; crítico; social; creativo, que contribuiría al desarrollo de la voluntad cubana de alcanzar un rol importante en el campo de la comunicación humana y social, a la vez que contribuir al perfeccionamiento de su imagen como nación con amplia capacidad

\footnotetext{
${ }^{1}$ Aquella cultura que ayuda a comprender y actuar críticamente en el contexto actual, para superar la desigualdad, y la dominación, como resultado de la experiencia vivida, cultura transformadora como vinculo resultante de la unión de la utopía con la realidad concreta necesitada de ser superada, operar este que favorece la participación de quienes más sienten la realidad social, lo que serviría como punto de conexión entre la acción educativa y la acción social.

${ }^{2}$ Aprendizaje que garantiza en el individuo la apropiación activa y creadora de la cultura, propiciando el desarrollo de su auto perfección constante, de su autonomía, en intima conexión con los necesarios procesos de socialización, compromiso y responsabilidad social.
} 
para el desarrollo y uso de las tecnologías de la información y la comunicaciones, en función de una sociedad prospera y sostenible.

\section{OBJETIVOS}

Explorar, describir y analizar el modo de incidir que tiene el uso de las TIC desde una concepción de empleo desarrolladora en la incorporación a la sociedad de sujetos en comunidades vulnerables mediante un proceso de Educación para la Comunicación consciente y planificado mediado por Joven Club de Computación y Electrónica.

\section{METODOLOGÍA}

Desde la perspectiva de la comunicación para el cambio social, la educación para la comunicación como práctica del Programa de Joven Club de Computación y Electrónica, podría contribuir significativamente al empoderamiento ciudadano, a la construcción participativa de ecosistemas comunicativos, a una mayor vinculación de los actores locales en función de potenciar su protagonismo comunitario y lograr una inclusión de estos sectores en el desarrollo local.

El presente informe, resultado de una investigación comunicológica, aborda el objeto de estudio, la educación para la comunicación ciudadana mediada por las TIC, desde una perspectiva epistemológica interpretativa. Es asumida por su capacidad para comprender la realidad como dinámica y diversa, y con un marcado interés en el significado de las acciones humanas y de la práctica social, caracterizándose por su relación investigador-objeto de estudio, la existencia de una participación democrática y comunicativa entre el investigador y los sujetos investigados. Considera la entrevista, la observación sistemática como técnicas de recopilación de información empírica para un estudio de caso como método primario de análisis de información.

El diseño de la investigación se corresponde con un estudio de caso único global descriptivo, dada su pertinencia para profundizar en el conocimiento de los procesos comunicativos y de construcción de competencias en un entorno vulnerable, y obtención de información relevante para la fundamentación de la propuesta. La selección del caso fue el resultado del análisis y discusión con los especialistas de la Dirección Provincial de los Joven Club en La Habana, quienes consideraron oportuno trabajar en el Palacio Central de la Computación: institución ubicada en el capitalino municipio de Centro Habana, al comportarse esta institución como el laboratorio de prueba de cualquier estrategia a implementar por la Dirección Nacional, además de contar con una infraestructura capaz de respaldar cualquiera de las acciones a desarrollar durante la investigación.

Desde el punto de vista de los razonamientos formulados estuvo presente el método del pensamiento Histórico -lógico, para la sistematización de las bases teóricoreferenciales de los principales núcleos conceptuales de la investigación; el Análisis -síntesis, para la comprensión de los fenómenos y elementos relacionados con el problema, la determinación de sus dimensiones e indicadores y el procesamiento de los resultados, a partir de la aplicación de los instrumentos; y el Inductivo- deductivo, 
para lograr abstracciones y generalizaciones necesarias que descubren las observancias y desacuerdos del proceso de educación para la comunicación tanto en los planos internacionales como nacionales y unificar criterios referidos a sus potencialidades para incidir en el desarrollo local, así como para identificar los puntos de ruptura y continuidad que existen entre el modelo cubano actuante cotejado y construido.

\section{DISCUSIÓN}

Es voluntad política de Cuba desarrollar la informatización de la sociedad, realidad que se evidencia actualmente en avances concretos. Este proceso que transversaliza las transformaciones ideológicas, económicas y sociales, es principio incuestionable del Gobierno, cuya principal prioridad es garantizar el acceso a las tecnologías de la información y la comunicación a la ciudadanía, poner la Internet al servicio de todos y lograr una inserción efectiva y auténtica de los cubanos en ese espacio.

El desarrollo de una política integral para el perfeccionamiento del proceso de informatización de la sociedad, aprobada por el consejo de ministro el 28 de febrero de 2017, se ha convertido en un arma para la defensa de esta voluntad de país, porque un conjunto de aspectos se han constituido en la razón de ser y prioridad del gobierno cubano, a saber: garantizar la Ciberseguridad frente a los riesgos y amenazas de todo tipo; asegurar la sostenibilidad y soberanía tecnológica; potenciar el acceso de los ciudadanos al empleo de las TIC; el desarrollo de los recursos tangibles; ensanchar y modernizar todas las esferas de la sociedad en apoyo a las prioridades del país; e integrar la investigación, el desarrollo y la innovación con la elaboración y comercialización de productos y servicios.

Para el logro y mantenimiento de estos resultados, es necesario la implementación y ejecución de un grupo de acciones encaminadas a preservar el desarrollo de la política de informatización de la sociedad cubana.

De ahí que sea motivo de la investigación mostrar el modo en que la virtualidad educativa que los medios digitales poseen, facilita el desarrollo de competencias comunicativas y participativas de las que están, especialmente, necesitados sujetos y grupos vulnerables, las cuales son parte esencial del desarrollo, siempre y cuando existan estrategias y prácticas institucionales que las potencien.

La propuesta se plantea explorar a las tecnologías de la información y la comunicación, desde su uso potenciador a partir de una determinada alternativa educativo-cultural: la educación transformadora (ET), entendiéndose como tal, aquella educación centrada en el aprendizaje del sujeto a partir de situaciones problémicas que generen la capacidad del individuo para el desarrollo de habilidades que lo capaciten para identificar y dar solución a situaciones de la vida práctica (Castellanos, 2000). En este sentido, no se trata sólo del modo de usar educativamente las TIC o de la manera de adaptarse a las nuevas realidades socioculturales, sino de convertir a los Joven Club de Computación y Electrónica en agentes culturales activos y transformadores, al insertarse en las redes sociales existentes, incluidas las locales, las virtuales y los movimientos sociales críticos 
como contribución al empoderamiento ciudadano que sirva de soporte al proceso de informatización de la sociedad cubana.

Al asumir la Educación para la Comunicación ciudadana como práctica y proceso educativo, destinado a permitir a los miembros de una comunidad participar de manera creativa y critica en la utilización de herramientas tecnológicas y tradicionales, a fin de contribuir a la formación de un sujeto comprometido, con capacidades para transformar su realidad, identificado con valores y comportamientos humanistas, éticos, de equidad social y justicia social, que contribuyan al desarrollo local (López, 2016a) y su contextualización en el modelo cubano, se observa que:

- Cuba ha identificado desde muy temprano la conveniencia y necesidad de dominar e introducir en la práctica social las Tecnologías de la Información y las Comunicaciones y lograr una cultura digital como una de las características imprescindibles del hombre nuevo, lo que facilitaría a nuestra sociedad acercarse más hacia el objetivo de un desarrollo sostenible.

- Un proyecto de desarrollo que tiene como pilares la justicia social, la participación popular, la equidad y la solidaridad, ha diseñado e iniciado la aplicación de estrategias que permiten convertir los conocimientos y las tecnologías de la información y las comunicaciones en instrumentos a disposición del avance y las profundas transformaciones revolucionarias.

- Un modelo de conceptualización del desarrollo económico que reconoce y promueve la implementación de políticas en función del desarrollo infotecnológico del país.

- Existencia de una política de Informatización de la Sociedad cubana que avala la transformación para el desarrollo de forma estructurada.

- Existencia de una política de comunicación que reconoce a la Comunicación Social como un importante componente del proceso de desarrollo económico y social como recurso estratégico de dirección del Estado y el Gobierno y las instituciones, las empresas, las organizaciones políticas y los medios de comunicación.

- Cuba ha identificado desde muy temprano la conveniencia y necesidad de dominar e introducir en la práctica social las Tecnologías de la Información y las Comunicaciones y lograr una cultura digital como una de las características imprescindibles del hombre nuevo, lo que facilitaría a nuestra sociedad acercarse más hacia el objetivo de un desarrollo sostenible.

- Un proyecto de desarrollo que tiene como pilares la justicia social, la participación popular, la equidad y la solidaridad, ha diseñado e iniciado la aplicación de estrategias que permiten convertir los conocimientos y las tecnologías de la información y las comunicaciones en instrumentos a disposición del avance y las profundas transformaciones revolucionarias.

- Un modelo de conceptualización del desarrollo económico que reconoce y promueve la implementación de políticas en función del desarrollo infotecnológico del país.

- Existencia de una política de Informatización de la Sociedad cubana que avala la transformación para el desarrollo de forma estructurada.

- Existencia de una política de comunicación que reconoce a la Comunicación Social como un importante componente del proceso de desarrollo económico y social como recurso estratégico de dirección del Estado y el Gobierno y las 
instituciones, las empresas, las organizaciones políticas y los medios de comunicación.

- La labor estratégica de los JCCE en el proceso de informatización de la sociedad.

- El Palacio Central de la Computación como espacio de confluencia y con una estructura e infraestructura acorde para la creación de una zona de confort desde su objeto social en el contexto municipal y barrial y la experiencia de la institución en el quehacer comunitario.

- Los Joven Club de Computación y Electrónica sirven de soporte informático para el desarrollo de otros programas sociales y culturales.

- La preparación de las nuevas generaciones en la utilización de las TIC y el empleo de éstas para aumentar la calidad del proceso docente educativo son elementos que buscan asegurar el futuro del país.

Todo lo anterior nos lleva a replantearnos los posibles desafíos para el contexto local:

- Posicionar a la Educación para la Comunicación como una herramienta fundamental para lograr una conciencia colectiva como contribución al cambio social.

- Aumentar el número de experiencias desde lo institucional en el uso de las TIC para lograr la formación de sujetos capaces de interactuar y usar los recursos de la comunicación desde el ejercicio de la crítica, la creatividad y la responsabilidad social.

- Lograr la articulación entre los actores que intervienen en el proceso de informatización de la sociedad cubana. (Educación, Cultura, Gobierno y JCCE).

- Incorporar contenidos esenciales en comunicación social en los planes de estudio en los diferentes niveles de enseñanzas y en la capacitación de los cuadros.

- Lograr el entendimiento por parte de los decisores sobre la comunicación como eje transversal del desarrollo y no solo como divulgación.

- Visibilizar JCCE como actor social fundamental en el proceso de cambio social, como mediador en el empoderamiento ciudadano desde su posición como medio de comunicación.

- Lograr una cultura comunicacional que facilite las posibilidades que desde el empoderamiento tribute a una calidad de vida del ciudadano.

- Establecer mecanismos de interacción Gobierno y el resto de los actores sociales, y la ciudadanía.

- Posicionar a la Educación para la Comunicación como una herramienta fundamental para lograr una conciencia colectiva como contribución al cambio social.

- Aumentar el número de experiencias desde lo institucional en el uso de las TIC para lograr la formación de sujetos capaces de interactuar y usar los recursos de la comunicación desde el ejercicio de la crítica, la creatividad y la responsabilidad social.

- Lograr la articulación entre los actores que intervienen en el proceso de informatización de la sociedad cubana. (Educación, Cultura, Gobierno y JCCE). 
- Incorporar contenidos esenciales en comunicación social en los planes de estudio en los diferentes niveles de enseñanzas y en la capacitación de los cuadros.

- Lograr el entendimiento por parte de los decisores sobre la comunicación como eje transversal del desarrollo y no solo como divulgación.

- Visibilizar JCCE como actor social fundamental en el proceso de cambio social, como mediador en el empoderamiento ciudadano desde su posición como medio de comunicación.

- Lograr una cultura comunicacional que facilite las posibilidades que desde el empoderamiento tribute a una calidad de vida del ciudadano.

- Establecer mecanismos de interacción Gobierno y el resto de los actores sociales, y la ciudadanía.

La referencia a una comunidad en condiciones de vulnerabilidad social llama necesariamente a la integración de todos los sectores socialmente vulnerables que forman dicha comunidad, incluyendo adultos mayores, niños y niñas, las personas con capacidades diferentes, desertores escolares, migrantes, entre otros, es por ello que este análisis nos lleva a crear mecanismo de regulación y control que permitan afrontar los desafíos y oportunidades en el campo de la educación para la comunicación que desde un modelo de Educación para la comunicación ciudadana ${ }^{3}$ prevé la integración de todos los actores locales en función.

Es por ello necesario la formación de un ciudadano a partir de la propuesta de Martínez de Toda (1998), para ayudar a la formación de un sujeto: alfabetizado mediáticamente; consciente; activo; crítico; social; creativo, que contribuiría al desarrollo de la voluntad cubana de alcanzar un rol importante en el campo de la comunicación humana y social, a la vez que contribuir al perfeccionamiento de su imagen como nación con amplia capacidad para el desarrollo y uso de las tecnologías de la información y la comunicaciones, en función de una sociedad prospera y sostenible.

Es apropiado expresar, que Joven Club de Computación y Electrónica se constituye en una alternativa asumida como soporte para la informatización de la sociedad cubana, por su condición de espacio de libre acceso a las nuevas tecnologías de la información y la comunicación, que dada su aceptación y el rol preponderante que asume como mediación en este proceso de formación, con un gran impacto en la reducción de la brecha digital, los convierten en la computadora de la familia cubana. Desde esta actuación se decide analizar, revisar y observar de manera sistemática la contribución de esta organización a la formación de un ciudadano capaz de interactuar con estos recursos tecnológicos, para ser utilizadas en el ejercicio de la crítica, la participación, la creatividad, como contribución al empoderamiento ciudadano.

El programa desde sus inicios ha mantenido firme la misión de: proporcionar una cultura informática a la comunidad con prioridad hacia niños y jóvenes, jugando un papel activo, creativo y de formación de valores en el proceso de informatización de la sociedad cubana.

\footnotetext{
${ }^{3}$ Investigación realizada por la autora en opción al grado científico de Doctor en Ciencias de la Comunicación diciembre de 2018 en la Universidad de La Habana.
} 
Desde los principios que la definen:

- Universalización

- Corresponsabilidad

- Participación

- Transversalidad

- Inclusión Social,

se ha constituido como institución a partir de tres líneas de actuación puntuales, que buscan dar respuesta a los objetivos trazados de acuerdo con los principios enumerados anteriormente y son ellas.

Línea de inserción Social y Laboral: pone a disposición de la población un conjunto de recursos de información útiles para la búsqueda de trabajo y la inserción al mundo laboral, además uno de los cursos que imparte esta institución es reconocido por el Ministerio del Trabajo y Seguridad Social, para garantizar el acceso a determinadas fuentes de trabajo (Curso de Operador de Microcomputadoras).

Línea de Uso Comunitario: es la línea que caracteriza más la filosofía de trabajo de la Institución. Ofrece espacios y soporte de accesibilidad para todos: personas individuales, entidades, grupos y colectivos.

Línea de Formación: esta línea es transversal a las otras dos líneas, dotándolas de soporte y consistencia. Desarrolla cursos de mediana y corta duración, talleres, círculos de interés, cursos de posgrado, entre otros, que permiten ofrecer itinerarios formativos más amplios y flexibles adecuados a la realidad y a la demanda de las personas y de la colectividad.

Como organización trabaja por lograr un mayor acceso a las TIC sobre la base de la socialización de los medios informáticos, el intercambio de información, la generalización de las experiencias más positivas y la accesibilidad total, apostando por el uso social, masivo e incluyente de la informática y la Internet, por encima del mercado y uso individual de estas tecnologías. Es una experiencia que permite que más cubanos accedan a este espacio para multiplicar su conocimiento y su cultura.

El desarrollo alcanzado por los Joven Club de Computación y Electrónica en materia de conectividad; la elevada preparación de sus técnicos y especialistas; la vasta experiencia en la prestación de servicios informáticos a la población cubana; la distribución de sus instalaciones a lo largo y ancho del territorio nacional y el alcance de su red de transmisión de datos, constituyen factores claves que propician la estructuración del servicio de acceso masivo de la sociedad cubana a la Red Cuba. A través de este programa, con treinta años de experiencia en trabajo comunitario, se objetivan las formas de mediación desde el uso de las TIC en la Educación para la Comunicación al contribuir a la formación de un sujeto activo, critico, comprometido, alfabetizado mediáticamente, identificado con valores y comportamientos humanistas que contribuyan al desarrollo local desde sus principios y líneas de actuación. En otras palabras, prácticas educomunicativa desde la visión de la Informática Comunitaria. 
Propuesta de modelo educomunicativo para el entorno local desde la mediación de los Joven Club.

\begin{tabular}{|c|c|c|}
\hline $\begin{array}{l}\text { Mecanismos y } \\
\text { Condiciones }\end{array}$ & Resultados previstos & Estrategias de operación \\
\hline $\begin{array}{l}\text { Oferta de formación } \\
\text { complementaria para el } \\
\text { desarrollo de competencias } \\
\text { básicas para un mejor } \\
\text { desempeño educativo y social. }\end{array}$ & $\begin{array}{l}\text {. Sujetos con capacidad de } \\
\text { ejercicio ciudadano } \\
\text { pertenecientes a cualquier } \\
\text { grupo etario. } \\
\text {. Sujetos con algún tipo de } \\
\text { discapacidad física, cognitiva. } \\
\text {. Organizaciones estatales y } \\
\text { privadas }\end{array}$ & $\begin{array}{l}\text { Validación y aplicación regular de espacios y } \\
\text { programas de formación complementaria. y/o } \\
\text { capacitación en el uso de las TIC para su } \\
\text { empoderamiento ciudadano. }\end{array}$ \\
\hline $\begin{array}{l}\text { Agenda comunitaria pro } \\
\text { entornos seguros para la } \\
\text { convivencia de niños/as, } \\
\text { adolescentes y jóvenes, con } \\
\text { participación intergeneracional }\end{array}$ & $\begin{array}{l}\text { Contexto de operación del } \\
\text { modelo con condiciones para } \\
\text { la convivencia y recreación de } \\
\text { los niños/as, adolescentes y } \\
\text { jóvenes, adultos y adultos } \\
\text { mayores }\end{array}$ & $\begin{array}{l}\text { Sensibilización y movilización de actores } \\
\text { locales para el diseño y ejecución de una } \\
\text { agenda comunitaria pro mejoramiento de la } \\
\text { seguridad y prevención de la violencia en el } \\
\text { entorno comunitario, con participación } \\
\text { intergeneracional desde el uso de las TIC. }\end{array}$ \\
\hline $\begin{array}{l}\text { Espacio donde operar el } \\
\text { modelo }\end{array}$ & $\begin{array}{l}\text { Espacio debidamente } \\
\text { equipado con infraestructura } \\
\text { para una interacción creativa y } \\
\text { constructiva de la población. }\end{array}$ & $\begin{array}{l}\text { Acondicionamiento de la infraestructura local } \\
\text { propia para la interacción social }\end{array}$ \\
\hline $\begin{array}{l}\text { Estructura de gestión operativa } \\
\text { responsable de la debida } \\
\text { implementación de los } \\
\text { mecanismos de intervención y } \\
\text { el logro de las metas acordadas }\end{array}$ & $\begin{array}{l}\text { La aplicación efectiva y } \\
\text { eficiente del modelo en todos } \\
\text { sus componentes }\end{array}$ & $\begin{array}{l}\text { Conformación, capacitación, } \\
\text { acompañamiento y supervisión del recurso } \\
\text { humano y técnico que integra la estructura de } \\
\text { gestión operativa del modelo. }\end{array}$ \\
\hline $\begin{array}{l}\text { Sistema de seguimiento y } \\
\text { valoración de resultados e } \\
\text { impacto, para determinar el } \\
\text { aporte del Modelo en sus } \\
\text { diversos elementos, en el } \\
\text { desarrollo local }\end{array}$ & $\begin{array}{l}\text { Identificación y sustentación } \\
\text { de los resultados e impacto de } \\
\text { la aplicación del modelo en el } \\
\text { contexto local. }\end{array}$ & $\begin{array}{l}\text { Aplicación de estrategias sistemáticas de } \\
\text { evaluación y rendición de cuentas ante los } \\
\text { actores locales. }\end{array}$ \\
\hline $\begin{array}{l}\text { Plataforma de Gestión } \\
\text { Estratégica desde el Gobierno } \\
\text { Local, con participación de los } \\
\text { actores locales para la } \\
\text { operación sostenible del } \\
\text { Modelo. }\end{array}$ & $\begin{array}{l}\text { Plataforma de Gestión } \\
\text { Estratégica de conformación } \\
\text { pública que garantiza los } \\
\text { recursos y supuestos de éxito } \\
\text { del Modelo }\end{array}$ & $\begin{array}{l}\text { Identificación y movilización del municipio y } \\
\text { otros actores locales clave, en la } \\
\text { consolidación de una plataforma de gestión } \\
\text { estratégica de los recursos y acciones que } \\
\text { requiere el Modelo para su efectividad y } \\
\text { sostenibilidad. }\end{array}$ \\
\hline
\end{tabular}

La concepción de trabajo de esta institución coincide plenamente con las dimensiones de la EPC, enunciados por Martínez de Toda y asumidas por la autora como condicionantes para el trabajo EPCC, pero desconocidas por los especialistas de los JCCE, lo que le resta calidad a sus servicios, los que se planifican de forma espontánea, como cumplimiento a las directivas que emite el órgano superior.

Una adecuada educación en comunicación, para desarrollar el análisis sobre medios y nuevas tecnologías de la comunicación, y contrariamente a los que han visto en ellos una solución didáctica en las escuelas y de entretenimiento en las familias, ha de: problematizar el contenido de los medios, para alejar la concepción

naturalizada que presentan de sí mismos; desideologizar sus mensajes, que tienden a legitimar y a reforzar "determinadas" actitudes, conductas e ideas sobre el mundo; evidenciar la articulación de los medios con el entramado 
comercial, empresarial, financiero y político para iluminar su pretendida «independencia y neutralidad (García, 2010).

La EPC contribuiría en gran medida a paliar situaciones como las antes referida, propiciando en la juventud posturas y estereotipos que sintonicen con las costumbres e idiosincrasia del país y no con los modelos marcados por los medios de comunicación masiva.

\section{CONCLUSIONES- RESULTADOS}

El papel de las TIC en la democracia local puede ser el motor de formas innovadoras de participación y gobierno que favorezcan la diversidad y calidad del trabajo comunitario, en el marco del desarrollo local.

El proceso de informatización de la sociedad se constituye en una alternativa para la inclusión social. Estas experiencias operan a medida de la cultura local y las necesidades de las comunidades.

Joven Club se constituye en una alternativa asumida como soporte en el proceso de informatización de la sociedad. Creado para proporcionar una cultura informática a la comunidad con prioridad hacia niños y jóvenes, jugando un papel creativo y de formación de valores.

La Educación para la Comunicación, en el marco de una enseñanza de calidad, tiene como función básica la formación de la conciencia crítica y el desarrollo de actitudes activas y creativas en sujetos para conocer y comprender los envolventes procesos de comunicación que vive la sociedad de hoy.

La Educación para la Comunicación ciudadana se constituye en práctica y proceso educativo, destinado a permitir a los miembros de una comunidad participar de manera creativa y critica en la utilización de herramientas tecnológicas y tradicionales, a fin de contribuir a la formación de un sujeto comprometido, con capacidades para transformar su realidad, identificado con valores y comportamientos humanistas, éticos, de equidad social y justicia social, que contribuyan al desarrollo local.

El modelo cubano de EPC se expresa a través de los actores locales, reconocidos en todo el país, lo componen estructuras de carácter nacional, provincial, municipal y barrial, en consecuencia con el contexto donde se desarrolle la experiencia, y a que decisores responde, cada uno de ellos caracterizados según cómo, donde, con quien y para quien se desarrolle el proceso, lo que evidencia la falta de una estructura, su realización depende en muchos casos de acciones de carácter empírico y espontáneas.

La investigación sobre el comportamiento de las prácticas de EPC en Cuba como parte de un proceso para el empoderamiento ciudadano de carácter universal, nos permite afirmar que la experiencia cubana carece de integración y se caracteriza por la dispersión e intermitencia en su desarrollo. Entre los aspectos más críticos podemos señalar la falta de claridad en los objetivos a largo plazo, la poca 
sostenibilidad y la no existencia de una línea estratégica que les aporte coherencia y visión de conjunto. A partir de la identificación de las potencialidades de este campo de la comunicación y su influencia directa en el desarrollo local se puede establecer una línea de ruptura entre el modelo actuante y el modelo propuesto para el uso de JCCE que puede incidir desde la transformación de un sujeto en riesgo de vulnerabilidad social en el desarrollo local.

El proceso de EPCc desde las dimensiones que lo transversalizan y con enfoque participativo en los procesos de transformación que vive la comunidad, correctamente encausado desde la aplicación de la estrategia de desarrollo municipal puede ser una herramienta poderosa para consolidar la participación ciudadana, al lograr desde sus pretensiones un sujeto más consciente, activo, crítico, social, creativo que puede influir en la transformación sociocultural de la localidad y por ende convertirse en un agente dinamizador de los procesos comunicación para el cambio social.

La EPCc en el actual contexto cubano, debe ser capaz de reconocer los cambios tanto de carácter comunicativo como social y, a partir de su carácter flexible actuar como un agente catalizador en el proceso de transformación de los sujetos como condición para asumir los cambios y ser participe en el escenario local de la ejecución de acciones en pro del desarrollo. 